\title{
Effectiveness of COVID-19 Vaccines in Patients Under Maintenance Hemodialysis
}

\author{
Waleed $\mathrm{H}$ Mahallawi ${ }^{\prime}$ \\ Nadir A Ibrahim' \\ Walaa A Mumena $\mathbb{D}^{2}$ \\ 'Medical Laboratory Technology \\ Department, College of Applied Medical \\ Sciences, Taibah University, Madinah, \\ Saudi Arabia; ${ }^{2}$ Department of Clinical \\ Nutrition, College of Applied Medical \\ Sciences, Taibah University, Madinah, \\ Saudi Arabia
}

Background: Several published data on the dialysis population showed that antibody levels decreased after COVID-19 vaccinations in comparison to the overall population. We aimed to illustrate the persistence of humoral response after receiving second dose of the Pfizer or AstraZeneca vaccines in patients under maintenance hemodialysis (HD).

Methods: A total of 119 adult patients on HD who were recruited from a single hemodialysis center in Madinah, Saudi Arabia. An enzyme-linked immunosorbent assay (ELISA) was utilized to measure the specific antibody response to the spike protein in the serum samples.

Results: Mean age of patients was $48.5 \pm 13.5$ years, while mean time since starting the renal dialysis was $5.09 \pm 5.29$ years. Blood samples were collected after $89.2 \pm 25.7$ days of receiving the second dose of the vaccines. A very high positive correlation between anti-S IgG antibodies in pre- and post-dialysis was found $\left(r_{s}=0.93, p<0.001\right)$. Additionally, there was a high positive correlation between anti-S IgG antibody collected at baseline and followup blood samples (30 days apart) $\left(r_{s}=0.82, p<0.001\right)$. Moreover, patients who received Pfizer had significantly higher mean change in anti-S IgG antibodies compared to patients who received AstraZeneca $(0.41 \pm 0.94$ vs $0.03 \pm 0.30$, respectively, $p=0.026)$.

Conclusion: The majority of the patients included in this study were able to yield an immune response to the vaccine after receiving the two doses. Persistence of IgG antibodies in the majority of the patients on HD in response to COVID-19 vaccines is encouraging in terms of continuing to vaccinate this category of patients in addition to monitoring them.

Keywords: SARS-CoV-2, maintenance hemodialysis, COVID-19 vaccines, chronic kidney disease

\section{Introduction}

The coronavirus 19 (COVID-19) pandemic led to a massive effect on health as well as the economy. Its fast spread in addition to appearance of several mutant variants has increased the burden on governments all over the world. ${ }^{1}$ The scale of COVID19 diverges from asymptomatic ${ }^{2}$ to multiorgan dysfunction, comprising several diseases such as acute respiratory distress syndrome as well as acute kidney injury. ${ }^{3}$

Patients with renal disease characterize an exceptional population due to their immunosuppressed situation in addition to distinctive exposures. ${ }^{4}$ Reasons for chronic kidney disease (CKD) differ worldwide. The primary causes, eventually leading to end-stage renal disease (ESRD), include: diabetes mellitus type 2 (30 $-50 \%$ ), type 1 diabetes mellitus (3.90\%), hypertension $(27.2 \%)$ and other diseases. ${ }^{5}$

As kidney function drops there appears to be poorer vaccine responsiveness. ${ }^{6}$ Several approaches have been implemented to develop responses to other vaccines
Correspondence: Waleed H Mahallawi Medical Laboratory Technology Department, College of Applied Medical Sciences, Taibah University, Madinah, Saudi Arabia

Email wmahallawi@taibahu.edu.sa 
such as those for hepatitis B and influenza A, for example using higher vaccine doses and adding adjuvants, or even recommending extra immunizations. ${ }^{7}$ Nevertheless, there is insufficient information obtainable on the efficacy of COVID-19 vaccination for HD patients, since huge vaccine trials omitted patients on dialysis. ${ }^{8}$

Observations on humoral immune response of HD patients to SARS-CoV-2 are not well documented. Patients with kidney failure are vulnerable to infection and commonly show less advantageous seroconversion reaction to vaccines. ${ }^{9}$ Patients on maintenance HD are at risk for SARS-CoV-2 infection. ${ }^{10}$

Individuals with obesity, diabetes mellitus and CKD comprise patients at high risk for SARS-CoV-2. ${ }^{11,12}$ Amongst CKD patients, those with a serious illness were greater in number than those inother groups with various comorbidities in addition to a compromised immune system. $^{13}$

A recent study showed that there has been a relationship between comorbidities and severe SARS-CoV-2 infection prevalence among CKD patients and that was up to $4 \% .{ }^{14}$ Therefore, the need to vaccinate this group of patients is of high priority to assure that they are protected from infection. Presently, Phase III studies using several vaccines, such as BNT162b2 (Pfizer) and ChAdOx1 (AstraZeneca), are being performed ${ }^{8}$

Comorbidity among patients undergoing dialysis is at high rates. Those patients have variable immunosuppression levels in addition to being much more likely to live in a state of permanent caution; therefore, they are at high risk of acquiring SARS-CoV-2 infection and also of mounting severe disease complications. ${ }^{15}$ Hemodialysis patients showed an increased case fatality rate (from 20 to $30 \%$ ). ${ }^{16}$ A recent study concluded that a third dose of Pfizer vaccine led to a considerable increase in antibody levels in dialysis patients, particularly in patients with suboptimal antibody levels following receipt of the second dose. ${ }^{17}$ Therefore, the Food and Drug Administration (FDA) is piloting an independent assessment of the safety as well as efficiency of a booster COVID-19 vaccine dose after receiving the first two doses. Moreover, the Advisory Committee on Immunization Practices (ACIP) will be providing booster dose endorsements based on a comprehensive evaluation of the data (https://www.cdc.gov/vaccines/covid-19/clinical-considera tions/covid-19-vaccines-us.html\#considerations-additionaldose, accessed on 20-09-2021).

Current evidence concerning the impact of hemodialysis on the humoral immune response is limited. Thus, we aimed in the current study to investigate two things: first, the responsiveness of maintenance HD patients to Pfizer and AstraZeneca vaccines; secondly, the humoral immune response following receipt of the two doses of COVID-19 vaccines in those patients.

\section{Materials and Methods Sample Collection}

This cohort study included all adult patients on maintenance HD from a single hemodialysis center in Madinah, Saudi Arabia $(\mathrm{n}=128)$. Exclusion criteria include not being vaccinated and receiving mixed doses of the vaccine (one dose Pfizer and one dose AstraZeneca). The study was conducted between January 1st and August 25th 2021. Data concerning each patient's age, sex, ethnicity, years of dialysis, frequency of dialysis per week, history of COVID-19 infection, vaccination status, and vaccine type were collected. In addition, $5 \mathrm{~mL}$ of blood was obtained from arterial port of the fistula during needling from the participants at different time points: baseline and followup (30 days apart). Following serum sample separation, they were reserved at $-70{ }^{\circ} \mathrm{C}$ for forthcoming antibody analysis. All participants were informed about the purpose of the study, in accordance with the Declaration of Helsinki. Signed consent forms were obtained from all patients included in this study. Ethical approval to conduct this study was obtained from the Research Ethics Committee of the College of Applied Medical Sciences, Taibah University (2021/101/212/MLT).

\section{Enzyme-Linked Immunosorbent Assay (ELISA)}

ELISA for the detection of SARS-CoV-2 anti-S IgG antibodies was performed using the commercial SARS-CoV-2 IgG ELISA Kit (BGI Europe A/S) according to the manufacturer's instructions. The test specificity for IgG antibody detection is $98.38 \%$, and the sensitivity for IgG antibody detection is greater than $98.71 \%$, according to the manufacturer. In brief, 96-well ELISA plates were used. Two wells of the plate were reserved for the negative control (for cut-off calculations) and one well contained the positive control and one well was left blank; $100 \mu \mathrm{L}$ of positive control and negative control were added to the designated wells without dilution. For the remaining wells, $10 \mu \mathrm{L}$ of the plasma was added to each well along with $100 \mu \mathrm{L}$ of sample diluent buffer, and the plates were incubated at $37^{\circ} \mathrm{C}$ for 30 minutes. The plates were then 
washed five times using a semi-automated ELISA washer, and $100 \mu \mathrm{L}$ of horseradish peroxidase-labeled anti-human IgG antibody was added to each well and incubated for 20 minutes at $37^{\circ} \mathrm{C}$. The plates were then washed five times, and $50 \mu \mathrm{L}$ each of substrates $\mathrm{A}$ and $\mathrm{B}$ were added to each well and incubated in the dark for 10 minutes at $37^{\circ} \mathrm{C}$. After the color development was finalised, $50 \mu \mathrm{L}$ of stopping solution was added to each well. Finally, the optical densities (ODs) were measured at $450 \mathrm{~nm}$. The cut-off value was calculated using the formula $0.1+$ mean absorbance of the two negative controls, and it was 0.18 .

\section{Statistical Analysis}

Data concerning level of anti-S IgG antibodies as well as age, time since receiving the second dose of COVID-19 vaccine, and time since starting renal dialysis are presented as mean \pm standard deviation (SD). Frequencies and percentages $(\%)$ are used to present data for categorical variables. The Shapiro-Wilk test was used to assess the normality of distribution of all continues variables. Spearman correlation was used to assess the association between the anti-S IgG antibodies pre- and post-dialysis and follow-up. The Mann-Whitney test was used to compare the mean of anti-S IgG antibody levels across two groups, while the Kruskal-Wallis test was used to compare the means across three groups or more. Simple linear regression analysis was used to investigate predictors of change in anti-S IgG antibody levels between baseline reading and last reading collected. Sensitivity analysis was performed after excluding patients with previous COVID-19 infection. SPSS software version 20 was utilized to analyse data presented in this study (IBM Corp., Armonk, N.Y., USA). All tests used were two-tailed and an alpha of 0.05 was used to assess the significance of associations tested.

\section{Results}

\section{Sample Characteristics}

A total of 119 patients were included in this study after excluding unvaccinated patients $(\mathrm{n}=7,5.47 \%)$ and patients who received mixed doses of the vaccine $(n=2$, $1.56 \%$ ). Mean age of patients was $48.5 \pm 13.5$ years, while mean time since starting renal dialysis was $5.09 \pm 5.29$ years. Blood samples were collected within $89.2 \pm 25.7$ days of receiving the second dose of the vaccine. Sixtyfour percent of the study sample was male $(n=76)$, with $47.1 \%(\mathrm{n}=56)$ Arabs, 29.4\% $(\mathrm{n}=35)$, South and East
Asian, and 23.5\% $(\mathrm{n}=28)$ African. Renal failure was reported to be caused by hypertension among the majority of the sample $(68.9 \%, \mathrm{n}=82)$. The majority of patients included in this study were on hemodialysis three time per week $(95.0 \%, \mathrm{n}=113)$. According to the $\mathrm{MOH}$ database (HESN) and patients self-reporting, most of the patients were not previously infected with SARS CoV2 (70.6\%, n $=84)$. The majority of patients were vaccinated with AstraZeneca $(83.2 \%, n=99)$. Seven percent $(n=8)$ of patients did not respond to the vaccine. Characteristics of patients included in this study are presented in Table 1.

Mean anti-S IgG antibodies in baseline blood samples was $2.42 \pm 1.10 \mathrm{OD}$ at $450 \mathrm{~nm}$, while mean anti-S IgG antibodies in follow-up blood samples was $2.52 \pm 1.02 \mathrm{OD}$ at $450 \mathrm{~nm}$. Mean change in anti-S IgG antibodies between baseline and follow-up blood samples was $0.09 \pm 0.49$ OD at $450 \mathrm{~nm}$.

\section{Correlations Between Anti-S IgG Antibodies Pre- and Post-Dialysis}

To assess the effect of renal dialysis on the antibody levels pre- and post-dialysis, we tested the participants' blood samples once during the study, at one session of dialysis. Findings of the Spearman correlation show very high positive correlations between anti-S IgG antibodies in pre- and post-dialysis blood samples collected $\left(r_{s}=0.93\right.$, $p<0.001)$. Additionally, to evaluate the stability and durability of anti-S IgG antibody levels after different time periods post-vaccination, we correlated the anti-S IgG antibodies collected at baseline and follow-up blood samples and found a high positive correlation $\left(\mathrm{r}_{\mathrm{s}}=0.82, p\right.$ $<0.001$ ); see Figures 1 and 2 .

Sensitivity analysis was performed and, after excluding patients with previous COVID-19 infection, similar results were obtained. Spearman correlations show very high positive correlations between the anti-S IgG antibody levels of pre- and post-dialysis blood samples collected $\left(\mathrm{r}_{\mathrm{s}}=0.95, p<0.001\right)$, while the correlation between the anti-S IgG antibodies collected at baseline and follow-up blood samples was highly positive $\left(\mathrm{r}_{\mathrm{s}}=\right.$ 0.83, $p<0.001)$.

This result could suggest the persistence of vaccineinduced anti-S IgG antibodies for a longer period in these patients, which would decrease concern regarding their fast decline because of frequent dialysis over the time period. 
Table I Characteristics of the Study Sample $(n=119)$

\begin{tabular}{|c|c|c|}
\hline & $\mathbf{n}$ & $\%$ \\
\hline \multicolumn{3}{|l|}{ Sex } \\
\hline Male & 76 & 63.9 \\
\hline Female & 43 & 36.1 \\
\hline \multicolumn{3}{|l|}{ Ethnicity } \\
\hline Arab & 56 & 47.1 \\
\hline African & 28 & 23.5 \\
\hline South and East Asian & 35 & 29.4 \\
\hline \multicolumn{3}{|l|}{ Cause of renal failure } \\
\hline Hypertension & 82 & 68.9 \\
\hline Diabetes & 5 & 4.20 \\
\hline Hypertension and diabetes & 29 & 24.4 \\
\hline Other diseases (eg heart disease) & 3 & 2.50 \\
\hline \multicolumn{3}{|l|}{ Frequency of hemodialysis } \\
\hline Twice per week & 6 & 5.04 \\
\hline Three times per week & 113 & 95.0 \\
\hline \multicolumn{3}{|l|}{ Previous diagnosis of COVID-19 } \\
\hline Yes & 35 & 29.4 \\
\hline No & 84 & 70.6 \\
\hline \multicolumn{3}{|l|}{ Type of COVID-19 vaccine } \\
\hline Pfizer & 20 & 16.8 \\
\hline AstraZeneca & 99 & 83.2 \\
\hline
\end{tabular}

\section{Associations Between Sample}

\section{Characteristics and Change in Anti-S IgG} Antibody Levels

Mean change in anti-S IgG antibodies in baseline and follow-up blood samples was similar among males and females $(0.10 \pm 0.55$ vs $0.08 \pm 0.38$, respectively; $p=$ $0.376)$. In addition, mean change in anti-S IgG antibodies in baseline and follow-up blood samples was similar among Arab, African, and South and East Asian participants $(0.10 \pm 0.51,0.03 \pm 0.16,0.13 \pm 0.62$, respectively; $p=0.789)$.

After excluding patients with previous COVID-19 infection, similar results were obtained. Mean change in anti-S IgG antibodies in baseline and follow-up blood samples was similar among males and females $(0.13 \pm$

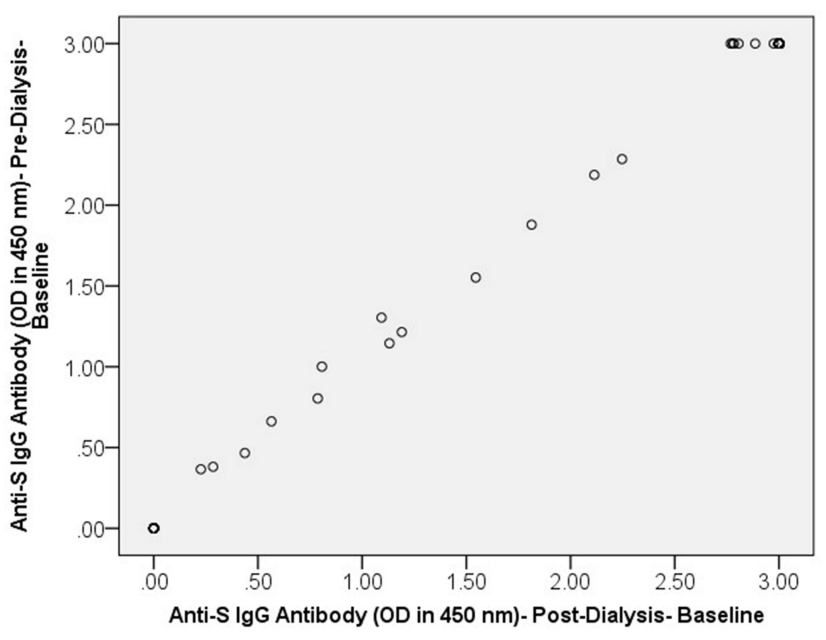

Figure I Correlations between anti-S IgG antibody levels pre- and post-dialysis.

0.62 vs $0.14 \pm 0.48$, respectively; $p=0.311$ ), whereas mean change in anti-S IgG antibodies in baseline and follow-up blood samples was similar among Arab, African, and South and East Asian participants (0.15 \pm $0.61,0.05 \pm 0.22,0.16 \pm 0.67$, respectively; $p=0.823)$.

Mean change in anti-S IgG antibodies in baseline and follow-up blood samples was similar in patients who were not diagnosed with COVID-19 compared to patients who were diagnosed with COVID-19 $(0.14 \pm 0.58$ vs $-0.00 \pm$ 0.03 , respectively; $p=0.337)$. Despite the persistence in the antibody levels of both vaccines, we found that patients who received Pfizer had significantly higher mean change in anti-S IgG antibodies compared to patients who received AstraZeneca $(0.41 \pm 0.94$ vs $0.03 \pm 0.30$, respectively; $p=0.026)$. After excluding patients with

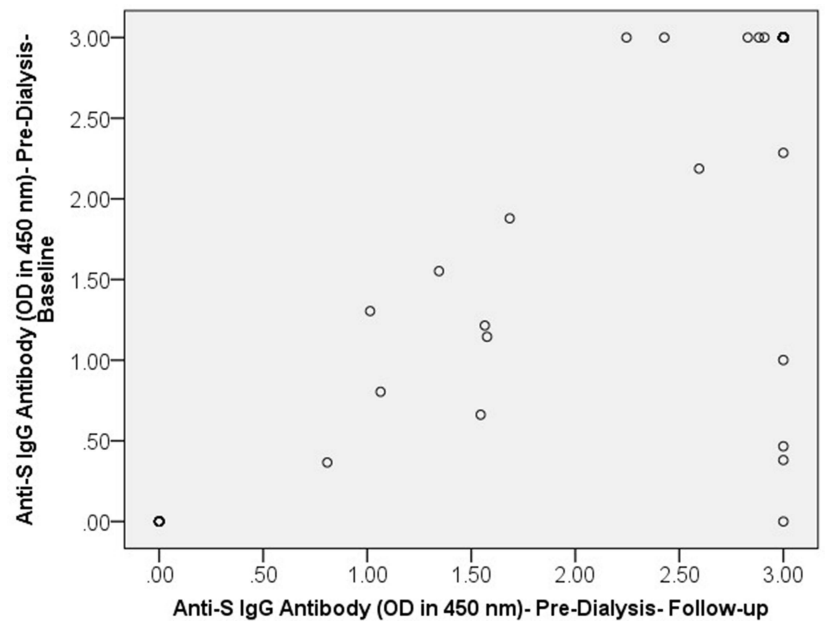

Figure 2 Correlations between anti-S IgG antibody levels pre-dialysis at baseline and follow-up. 
previous COVID-19 infection, a similar result was found whereby patients who received Pfizer had significantly higher mean change in anti-S IgG antibodies compared to patients who received AstraZeneca $(0.51 \pm 1.04$ vs 0.05 \pm 0.36 , respectively; $p=0.038$ ).

No correlation was found between change in anti-S IgG antibodies in baseline and follow-up blood samples and time since receiving the second dose of COVID-19 vaccine $\left(\mathrm{r}_{\mathrm{s}}=-0.01, p=0.911\right)$. This result is also of interest as it proves the sustainability of antibody at higher levels even a long time after receiving the two vaccine doses.

\section{Predictors of Change in Anti-S IgG Antibody}

Simple linear regression analysis was performed to investigate predictors of change in anti-S IgG antibody. Participants' age, sex, ethnicity, time since starting renal dialysis, and previous diagnosis of COVID-19 did not predict changes in anti-S IgG antibodies. In fact, the use of Pfizer vaccine predicted increased anti-S IgG antibodies $(\mathrm{B}=-0.38, \mathrm{SE}=0.12$ [95\% CI: -0.61 to -0.15$]$, R-square $=0.08$ ), while longer time since receiving the second dose of COVID-19 vaccine predicted lower anti-S IgG antibodies ( $\mathrm{B}=-0.004, \mathrm{SE}=0.002$ [95\% CI: -0.007 to -0.001 ], R-square $=0.04$ ); see Table 2 .

After excluding patients with previous COVID-19 infection. similar results were obtained. Only the use of Pfizer vaccine predicted increased anti-S IgG antibodies $(\mathrm{B}=-0.47, \mathrm{SE}=0.15$ [95\% CI: -0.77 to -0.16$], p=0.003$, $\mathrm{R}$-square $=0.10$ ), and longer time since receiving the second dose of COVID-19 vaccine predicted lower anti$\mathrm{S}$ IgG antibodies $(\mathrm{B}=-0.005, \mathrm{SE}=0.002[95 \% \mathrm{CI}$ : -0.009 to -0.001 ], $p=0.029$, R-square $=0.06$ ).

\section{Discussion}

Undesirably, responsiveness to immunization in patients suffering from renal disease can be weakened due to the changes in the function of the immune system; therefore, they are described as members of an increased risk population. ${ }^{18}$ The key goal of the COVID-19 vaccine is to create immunity to the viral spike protein of the virus. Vaccine-mediated immunity to the SARS-CoV-2 is generated to prevent the interaction between its spike protein and angiotensin-converting enzyme 2 (ACE-2). ${ }^{19}$ Patients with kidney diseases should be selected for COVID-19 vaccination and the existing information recommends that replication-defective viral-vectored and mRNA vaccines are safe for administration to this population. ${ }^{20}$

Findings of the present study are in line with those of previous studies showing that patients on maintenance HD mount a humoral immune response following COVID-19 vaccines. ${ }^{21}$ Our cohort in the current study received two doses of either BNT162b2 (Pfizer) or ChAdOx1 (AstraZeneca). The first is an mRNA-based vaccine, a lipid nanoparticlecapture that encodes perfusion-stabilized full-length spike proteins. ${ }^{22}$ The latter is a vector vaccine that uses the full-length codon for the S-protein. ${ }^{23}$ The main finding from our study is that a great percentage of our patients were able to yield an immune response to these two vaccines. Additionally, we showed that Pfizer vaccine is superior to AstraZeneca in terms of maintaining higher anti-S IgG antibodies. We also found that all the respondent patients $(n=95)$ maintained detectable anti-S IgG antibodies at higher levels (from 1.02 to 3, with a mean of 2.85 OD units). This result aligns with a study conducted by Grupper et al, who found that a higher percentage of patients on dialysis maintained higher titrations of anti-S antibodies after the second dose of the

Table 2 Predictors of Changes in Anti-S IgG Antibodies in Baseline and Follow-Up Blood Samples Among Vaccinated Renal Failure Patients on Hemodialysis

\begin{tabular}{|l|c|c|c|c|c|}
\hline & B & SE & $\boldsymbol{P}$ & $\mathbf{9 5 \%}$ Confidence Interval & R-Square \\
\hline Age, years & -0.01 & 0.00 & 0.108 & -0.01 to 0.00 & 0.02 \\
\hline Sex (male = I; female = 2) & -0.02 & 0.09 & 0.834 & -0.21 to 0.17 & 0.00 \\
\hline Ethnicity (Arab = I; African = 2; South and East Asian = 3) & 0.01 & 0.05 & 0.874 & -0.10 to 0.11 & 0.00 \\
\hline Time since starting renal dialysis, years & 0.00 & 0.01 & 0.857 & -0.02 to 0.02 & 0.00 \\
\hline Type of COVID-19 vaccine (Pfizer = I; AstraZeneca = 2) & -0.38 & 0.12 & $0.00 I^{*}$ & -0.61 to -0.15 & 0.08 \\
\hline Time since receiving the second dose of COVID-19 vaccine, days & -0.004 & 0.002 & $0.023^{*}$ & -0.007 to -0.001 & 0.04 \\
\hline
\end{tabular}

Note: $*$ Alpha $=0.05$ 
Pfizer vaccine. ${ }^{24}$ Additionally, a recent study showed that vaccine-induced humoral immune response caused seroconversion efficacy ( $>95 \%$ ) in dialysis patients that was analogous to medical personnel. ${ }^{25}$ Moreover, a recent study also is in agreement with ours but they also measured the neutralizing activity as they showed $82 \%$ of the patients on dialysis developed neutralizing antibodies after the second dose. ${ }^{26}$

This result gives a very positive message as evidence of the efficiency of the vaccines and sustainability of the vaccine-induced humoral immune response. Our result is in agreement with a recent work on comparing humoral response to mRNA and an adenovirus vector-based SARSCOV2 (Ad26.COV2.S) vaccine in dialysis patients, which suggested consideration of the former vaccines over the latter for dialysis patients because of their prolonged higher antibody levels for a longer time. ${ }^{27}$

Our results could suggest the persistence of vaccineinduced anti-S IgG antibodies for a longer period in these patients, which would decrease concern regarding their fast decline because of frequent dialysis over the time period. It is worth noting that almost $98 \%$ of the patients were scheduled to attend the renal dialysis center three times per week. Despite this, they maintained higher antibody levels even though there was also a long time period between receiving the second vaccine dose and our study sampling.

Unfortunately, we found that $8.26 \%(n=10)$ of the vaccinated patients included in this study were nonresponders and had undetectable antibodies. All the nonresponders $(10.1 \%, \mathrm{n}=10)$ received AstraZeneca vaccine. Data from the mRNA vaccine trials propose a strong antibody response is retained for up to three months, but we have no data beyond this in the general population and no data beyond 30 days in patients on dialysis. ${ }^{1}$ However, our data show persistence of antibodies for more than four months after receiving the second dose.

Hypertension was the major cause of our patients' renal failure (90.6\%); they were either hypertensive-only $(66.4 \%)$ or hypertensive and diabetic (24.2\%) simultaneously. Hypertension and diabetes are risk factors for SARS-CoV-2 infection. Additionally, hypertension and diabetes mellitus are common comorbid conditions that accounte for the high risk factor in COVID-19 disease severity and mortality. ${ }^{28}$ Several predictive factors have been associated with COVID-19 severity, such as weakened immune response and raised inflammatory response. $^{29}$ Unfortunately, $8 \%$ of the vaccinated patients involved in our study were non-responders and had undetectable antibodies. Therefore, we suggest that more boosting or a higher dose could be investigated in those nonresponders; alternatively, other administration routes for vaccinations could be considered, such as an intranasal vaccine. ${ }^{30,31}$ Whether IgG antibodies last and lead to protective immunity against future SARS-CoV-2 infection needs more investigation.

Causes of seroconversion failure in dialysis patients indicate a need for immune monitoring as well as adjustment of vaccination practices. A complete understanding of the vaccination-induced modifications in SARS-CoV-2 specific immunity can allow substitute vaccination strategy or improvement of new vaccine formations. Consequently, a comprehensive study of SARS-CoV-2 cellular immune response is needed to get a conclusion about vaccine-derived immunity in renal dialysis patients. Worries raised by the health authority over the antibody waning in patients who are under prolonged renal dialysis concern these patients. Therefore, recommendations from scientific advisory organizations, such as the WHO, CDC and European Medicines Agency, should be followed regarding vaccinating those patients at high risk from the SARS-COV2. Authorized COVID-19 vaccines offer a great amount of protection from becoming extremely ill or indeed dying from the disease (https://www.cdc.gov/coronavirus/2019-ncov/vaccines/ recommendations-process.html, https:/www.who.int/emer gencies/diseases/novel-coronavirus-2019/covid-19-vac cines/advice and https:/www.ema.europa.eu/en/news/emaecdc-update-covid-19, accessed 20-09-2021).

We showed that there were no associations between anti-S IgG antibody levels and sex or ethnicity of patients. Additionally, the mean change in anti-S IgG antibodies in baseline and follow-up blood samples was similar in patients who were not diagnosed with COVID-19 and patients who were diagnosed with COVID-19. However, this result is in opposition to studies showing that people with prior COVID-19 developed higher humoral immunity after receiving the vaccines; however, those studies were conducted on healthy individuals. ${ }^{32,33}$ Therefore, more studies on hemodialysis patients are required to give a conclusion about this phenomenon.

Due to the weakened immune response of patients having renal dialysis, monitoring of the longevity of vaccine-induced antibodies against several infectious diseases is needed. For example, the median response percentage for Hepatitis B virus vaccine is $64 \%$ with the 3 -dose plan in comparison with up to $95 \%$ with healthy people ${ }^{34}$. The 
ACIP recommends vaccination of one dose of the 23valent pneumococcal vaccine for patients with $\mathrm{CKD}{ }^{35}$ While patients with renal disease retained a lessened immunologic response, fruitful immunization of them is likely, which will reduce the possibility of problems from vaccine-preventable illnesses. ${ }^{36}$

Presently, in the absence of randomized trials, no optimum vaccination strategy in CKD patients is existing. Moreover, several policies are applied to heighten the vaccine-induced seroconversion proportion in progressive CKD patients. Extra investigation is necessary to expand magnitudes of sero responsiveness. However, obedience to the offered vaccination recommendations is extremely necessary in these immunocompromised patients.

Our study is the first to investigate the effect of HD on IgG antibody levels among a relatively large and multiethnic sample. However, our study has some limitations. First, humoral response to the vaccines was evaluated in a single-center small-sized cohort, which restricted statistical analyses and might cause bias. Second, it is limited by the lack of sequential blood samples taken after the participants received the first and second vaccine doses. Third, data on vaccine-induced cellular immunity were not investigated. Fourth, samples included at baseline were not collected after the second vaccine dose at a fixed time (2-3 weeks). Fifth, we did not compare our results to those of a control group. Lastly, because of the lack of facilities, we could not perform the neutralization assay for the samples. Finally, as our study is observational, there is an inconsistency in the time between the receiving of the second dose and antibody measurement, which might be a possible cause of bias.

In conclusion, the majority of the patients included in this study were able to yield an immune response to the vaccine after receiving the two doses. Persistence of IgG antibodies in the majority of the patients on $\mathrm{HD}$ in response to COVID-19 vaccines is encouraging in terms of continuing to vaccinate this category of patients in addition to monitoring them.

\section{Acknowledgments}

The authors extend their appreciation to Taibah University, represented by the Deanship of Scientific Research, for funding this project (no. RC-442/4). We also thank Hayat Organization for sponsoring all of the hemodialysis patients who were recruited in the study.

\section{Disclosure}

The authors have no affiliation with any organization with a direct or indirect financial interest in the subject matter discussed in this article. The authors report no conflicts of interest in this work.

\section{References}

1. Zhu N, Zhang D, Wang W, et al. A novel coronavirus from patients with pneumonia in China, 2019. N Engl J Med. 2020;382(8):727733. doi:10.1056/NEJMoa2001017

2. Mahallawi WH, Ibrahim NA, Aljohani AS, et al. Assessment of SARS-CoV-2 anti-spike IgG antibody in women and children in Madinah, Saudi Arabia: a single-center study. Int J Environ Res Public Health. 2021;18(19):9971. doi:10.3390/ijerph18199971

3. Hou YC, Lu KC, Kuo KL. The efficacy of COVID-19 vaccines in chronic kidney disease and kidney transplantation patients: a narrative review. Vaccines (Basel). 2021;9(8). doi:10.3390/vaccines9 080885

4. Collins AJ, Foley RN, Gilbertson DT, Chen S-C. United States Renal Data System public health surveillance of chronic kidney disease and end-stage renal disease. Kidney Int Suppl. 2015;5(1):2-7. doi:10.1038/kisup. 2015.2

5. Webster AC, Nagler EV, Morton RL, Masson P. Chronic kidney disease. Lancet. 2017;389(10075):1238-1252. doi:10.1016/S01406736(16)32064-5

6. Krueger KM, Ison MG, Ghossein C. Practical guide to vaccination in all stages of $\mathrm{CKD}$, including patients treated by dialysis or kidney transplantation. Am J Kidney Dis. 2020;75(3):417-425. doi:10.1053/ j.ajkd.2019.06.014

7. Schulte K, Schierke H, Tamayo M, et al. Strategies for improving influenza vaccination rates in patients with chronic renal disease. Dtsch Arztebl Int. 2019;116(23-24):413-419. doi:10.3238/ arzteb1.2019.0413

8. Glenn DA, Hegde A, Kotzen E, et al. Systematic review of safety and efficacy of COVID-19 vaccines in patients with kidney disease. Kidney Int Rep. 2021;6(5):1407-1410. doi:10.1016/j. ekir.2021.02.011

9. Soni R, Horowitz B, Unruh M. Immunization in end-stage renal disease: opportunity to improve outcomes. Semin Dial. 2013;26 (4):416-426. doi:10.1111/sdi.12101

10. Chan L, Fuca N, Zeldis E, Campbell KN, Shaikh A. Antibody response to mRNA-1273 SARS-CoV-2 vaccine in hemodialysis patients with and without prior COVID-19. Clin J Am Soc Nephrol. 2021;16:1258-1260. doi:10.2215/CJN.04080321

11. Alam MR, Kabir MR, Reza S. Comorbidities might be a risk factor for the incidence of COVID-19: evidence from a web-based survey. Prev Med Rep. 2021;21:101319. doi:10.1016/j.pmedr.2021.101319

12. Huang C, Soleimani J, Herasevich S, et al. Clinical characteristics, treatment, and outcomes of critically ill patients with COVID-19: a scoping review. Mayo Clin Proc. 2021;96(1):183-202. doi:10.1016/j. mayocp.2020.10.022

13. Kant S, Menez SP, Hanouneh M, et al. The COVID-19 nephrology compendium: AKI, CKD, ESKD and transplantation. BMC Nephrol. 2020;21(1):449. doi:10.1186/s12882-020-02112-0

14. Singh AK, Gillies CL, Singh R, et al. Prevalence of co-morbidities and their association with mortality in patients with COVID-19: a systematic review and meta-analysis. Diabetes Obes Metab. 2020;22 (10):1915-1924. doi:10.1111/dom.14124

15. Weinhandl ED, Wetmore JB, Peng Y, Liu J, Gilbertson DT, Johansen KL. Initial effects of COVID-19 on patients with ESKD. J Am Soc Nephrol. 2021;32(6):1444-1453. doi:10.1681/ ASN.2021010009 
16. Taji L, Thomas D, Oliver MJ, et al. COVID-19 in patients undergoing long-term dialysis in Ontario. CMAJ. 2021;193(8):E278-e84. doi:10.1503/cmaj.202601

17. Bensouna I, Caudwell V, Kubab S, et al. SARS-CoV-2 antibody response after a third dose of the BNT162b2 vaccine in patients receiving maintenance hemodialysis or peritoneal dialysis. $\mathrm{Am} \mathrm{J}$ Kidney Dis. 2021. doi:10.1053/j.ajkd.2021.08.005

18. Dinits-Pensy M, Forrest GN, Cross AS, Hise MK. The use of vaccines in adult patients with renal disease. Am J Kidney Dis. 2005;46 (6):997-1011. doi:10.1053/j.ajkd.2005.08.032

19. Scialo F, Daniele A, Amato F, et al. ACE2: the major cell entry receptor for SARS-CoV-2. Lung. 2020;198(6):867-877. doi:10.1007/ s00408-020-00408-4

20. Windpessl M, Bruchfeld A, Anders H-J, et al. COVID-19 vaccines and kidney disease. Nat Rev Nephrol. 2021;17(5):291-293. doi:10.1038/s41581-021-00406-6

21. Shaikh A, Zeldis E, Campbell KN, Chan L. Prolonged SARS-CoV-2 viral RNA shedding and IgG antibody response to SARS-CoV-2 in patients on hemodialysis. Clin J Am Soc Nephrol. 2021;16(2):290292. doi: $10.2215 /$ CJN. 11120720

22. Polack FP, Thomas SJ, Kitchin N, et al. Safety and efficacy of the BNT162b2 mRNA Covid-19 vaccine. $N$ Engl J Med. 2020;383 (27):2603-2615. doi:10.1056/NEJMoa2034577

23. Folegatti PM, Ewer KJ, Aley PK, et al. Safety and immunogenicity of the ChAdOx1 $\mathrm{nCoV}-19$ vaccine against SARS-CoV-2: a preliminary report of a phase $1 / 2$, single-blind, randomised controlled trial. Lancet. 2020;396(10249):467-478. doi:10.1016/S0140-6736(20)31604-4

24. Grupper A, Sharon N, Finn T, et al. Humoral response to the Pfizer BNT162b2 vaccine in patients undergoing maintenance hemodialysis. Clin J Am Soc Nephrol. 2021;16:1037-1042. doi:10.2215/ CJN.03500321

25. Stumpf J, Siepmann T, Lindner T, et al. Humoral and cellular immunity to SARS-CoV-2 vaccination in renal transplant versus dialysis patients: a prospective, multicenter observational study using mRNA1273 or BNT162b2 mRNA vaccine. Lancet Region Health. 2021;9:100178. doi:10.1016/j.lanepe.2021.100178

26. Speer C, Göth D, Benning L, et al. Early humoral responses of hemodialysis patients after COVID-19 vaccination with BNT162b2. $J$ Am Soc Nephrol. 2021;16(7):1073-1082. doi:10.2215/ CJN.03700321
27. Mulhern J, Fadia A, Patel R, et al. Humoral response to mRNA versus an adenovirus vector-based SARS-COV2 (Ad26.COV2.S) vaccine in dialysis patients. Clin J Am Soc Nephrol. 2021;16:17201722. doi: $10.2215 /$ CJN.06450521

28. Sun Y, Guan X, Jia L, et al. Independent and combined effects of hypertension and diabetes on clinical outcomes in patients with COVID-19: a retrospective cohort study of Huoshen Mountain Hospital and Guanggu Fangcang Shelter Hospital. J Clin Hypertens (Greenwich). 2021;23(2):218-231. doi:10.1111/jch.14146

29. Magdy Beshbishy A, Oti VB, Hussein DE, et al. Factors behind the higher COVID-19 risk in diabetes: a critical review. Front Public Health. 2021;9:637. doi:10.3389/fpubh.2021.591982

30. Rincon-Arevalo H, Choi M, Stefanski AL, et al. Impaired humoral immunity to SARS-CoV-2 BNT162b2 vaccine in kidney transplant recipients and dialysis patients. Sci Immunol. 2021;6:60. doi:10.1126/ sciimmunol.abj1031

31. Mahallawi WH, Aljeraisi TM. Infection with SARS-CoV-2 primes immunological memory in human nasal-associated lymphoid tissue. Clin Immunol. 2021;231:108850. doi:10.1016/j.clim.2021.108850

32. Abbasi J. Study suggests lasting immunity after COVID-19, with a big boost from vaccination. JAMA. 2021;326(5):376-377. doi:10.1001/jama.2021.11717

33. Jeyanathan M, Afkhami S, Smaill F, Miller MS, Lichty BD, Xing Z. Immunological considerations for COVID-19 vaccine strategies. Nat Rev Immunol. 2020;20(10):615-632.

34. Alter MJ, Arduino MJ, Lyerla HC, Miller ER, Tokars JI. Recommendations for preventing transmission of infections among chronic hemodialysis patients. MMWR Recomm Rep. 2001;50(Rr5): $1-43$.

35. Atkinson WL, Pickering LK, Schwartz B, Weniger BG, Iskander JK, Watson JC. General recommendations on immunization. Recommendations of the Advisory Committee on Immunization Practices (ACIP) and the American Academy of Family Physicians (AAFP). MMWR Recomm Rep. 2002;51(Rr-2):1-35.

36. Haddiya I. Current knowledge of vaccinations in chronic kidney disease patients. Int $J$ Nephrol Renovasc Dis. 2020;13:179-185. doi:10.2147/IJNRD.S231142
Risk Management and Healthcare Policy

\section{Publish your work in this journal}

Risk Management and Healthcare Policy is an international, peerreviewed, open access journal focusing on all aspects of public health, policy, and preventative measures to promote good health and improve morbidity and mortality in the population. The journal welcomes submitted papers covering original research, basic science, clinical \& epidemiological studies, reviews and evaluations, guidelines, expert opinion and commentary, case reports and extended reports. The manuscript management system is completely online and includes a very quick and fair peer-review system, which is all easy to use. Visit http://www.dovepress.com/testimonials.php to read real quotes from published authors. 